\title{
Wireless Power Transfer in Two-Way AF Relaying networks
}

DOI:

10.1109/GLOCOM.2016.7842389

\section{Document Version}

Accepted author manuscript

Link to publication record in Manchester Research Explorer

\section{Citation for published version (APA):}

Salem, A., \& Hamdi, K. (2017). Wireless Power Transfer in Two-Way AF Relaying networks. In Global

Communications Conference (GLOBECOM), 2016 IEEE IEEE. https://doi.org/10.1109/GLOCOM.2016.7842389

\section{Published in:}

Global Communications Conference (GLOBECOM), 2016 IEEE

\section{Citing this paper}

Please note that where the full-text provided on Manchester Research Explorer is the Author Accepted Manuscript or Proof version this may differ from the final Published version. If citing, it is advised that you check and use the publisher's definitive version.

\section{General rights}

Copyright and moral rights for the publications made accessible in the Research Explorer are retained by the authors and/or other copyright owners and it is a condition of accessing publications that users recognise and abide by the legal requirements associated with these rights.

\section{Takedown policy}

If you believe that this document breaches copyright please refer to the University of Manchester's Takedown Procedures [http://man.ac.uk/04Y6Bo] or contact uml.scholarlycommunications@manchester.ac.uk providing relevant details, so we can investigate your claim.

\section{OPEN ACCESS}




\title{
Wireless Power Transfer in Two-Way AF Relaying networks
}

\author{
Abdelhamid Salem and Khairi A. Hamdi \\ School of Electrical \& Electronic Engineering, \\ The University of Manchester, Manchester, UK \\ emails: \{abdelhamid.salem, k.hamdi\}@ manchester.ac.uk
}

\begin{abstract}
In this paper, we study a wireless-powered communication network (WPCN) scenario, in which a multiple-antenna amplify-and-forward (AF) two-way relay coordinates power transfer and information exchange to multiple pairs of users. A harvestthen-transmit protocol is assumed where the relay first transmits energy signals to the users in a power transfer phase. Multi-pair of users, which have rechargeable batteries, then use the harvested energy to exchange their independent signals through the relay in two phases, up-link (UL) and down-link (DL). In the UL phase, all the users transmit their information signals to the relay and in the DL phase the relay amplifies and forwards the signals to their intended users. Furthermore, in order to cancel out the interference, zero-forcing reception and transmission (ZFR/ZFT) is implemented at the relay. To characterize system performance, we consider ergodic spectral and energy efficiencies for two cases, based on the availability of the channel state information (CSI) at the relay during the power transfer phase, namely, 1) unknown CSI 2) partially known CSI. In light of this, we derive new analytical expressions for the ergodic spectral and energy efficiencies for the two cases and Monte Carlo simulations are provided throughout our investigations to validate the mathematical analysis. The impacts of some system parameters such as $\mathbf{E H}$ time, $\mathbf{E H}$ efficiency, number of relay antennas and user-pairs, on the system performance are investigated.
\end{abstract}

Index Terms-Multi-pair, zero forcing, wireless power transfer, spectral efficiency, two-way relaying, zero-forcing.

\section{INTRODUCTION}

$\mathbf{R}$ ADIO frequency $(\mathrm{RF})$ energy harvesting $(\mathrm{EH})$ in wireless communications has received significant attention in recent years. This technology is based on the fact that RF signals can carry energy. Therefore, energy constrained nodes can harvest energy from the RF signals transmitted by the source node [1]-[5]. This solution, becomes an appealing in applications where battery-limited devices are not easily accessible, and replacing or recharging their batteries is inconvenient such as devices embedded inside human bodies and sensors placed in dangerous areas. Recently, many researchers have investigated the performance of wireless-powered communication networks (WPCNs), where the RF signals are used to charge the energy constrained nodes. For instance, in [1], the tradeoff between the rates at which energy and reliable information can be transmitted over a single noisy channel was studied. Later on, this work was extended in [2] to incorporate frequency-selective channels with additive white Gaussian noise.

Energy transfer efficiency is subject to channel fading, similar to wireless information signals, and therefore, multi-antenna and cooperative communication techniques can be used to further increase the efficiency of such systems [6], [7]. For example, the authors in [8] studied the performance of energy beamforming in multiple-antennas WPCN. The system considered in [8] consists of a powered access-point (AP) equipped with multiple antennas and a user with single antenna. Furthermore, in [9] harvest-then-cooperate (HTC) protocol is proposed, where in the down-link the source and relay nodes harvest energy from the AP and in the up-link the two nodes work cooperatively for information transmission. A multiple energy-constrained source nodes with one fully-powered access point (AP) scenario was investigated in [10], [11] where the AP transfers power to the source nodes in phase I, down-link (DL), and then the source nodes use the harvested power to transmit their information to the AP in phase II, up-link (UL). This protocol is referred to as harvest-then-transmit. The work in [11] which considered singleantenna AP was later extended in [12] to include a multipleantenna AP.

To the best of the authors knowledge, multi-pair energyconstrained nodes with a fully-powered relaying AP scenario has not been studied in the literature yet. Therefore, in this paper, we analyze the performance of a two-way cooperative communication network consisting of multi-pair single-antenna users communicating via a two-way multiple-antenna relay. The relay is connected to a constant power supply whereas the users are $\mathrm{EH}$ nodes equipped with rechargeable batteries to store the harvested energy transmitted by the relay. The EH protocol considered here is the harvest-then-transmit protocol. The proposed system has three distinct phases

1) Phase I: (DL power transfer) the relay broadcasts $R F$ signals, the energy of which is extracted by all users to recharge their batteries. The harvested energy is then used to establish communications between the users via the relay during the remaining two phases.

2) Phase II: (UL information transfer) all users transmit their independent signals to the relay.

3) Phase III: (DL information transfer) the relay amplifies and forwards the received signals to their intended destinations.

Such scenario could occur in ad-hoc networks where powerconstrained multi-pair of users intend to establish a bidirectional transmission links via a relay node. Another example, where power-constrained sensor nodes exchange their measurements with each other in the sensor networks. In order to cancel out the interference and improve the system performance, zeroforcing reception and zero-forcing transmission (ZFR/ZFT) is implemented at the relay [13]. We adopt the ergodic spectral and energy efficiencies as performance measures to characterize system performance. The ergodic spectral and energy efficiencies 


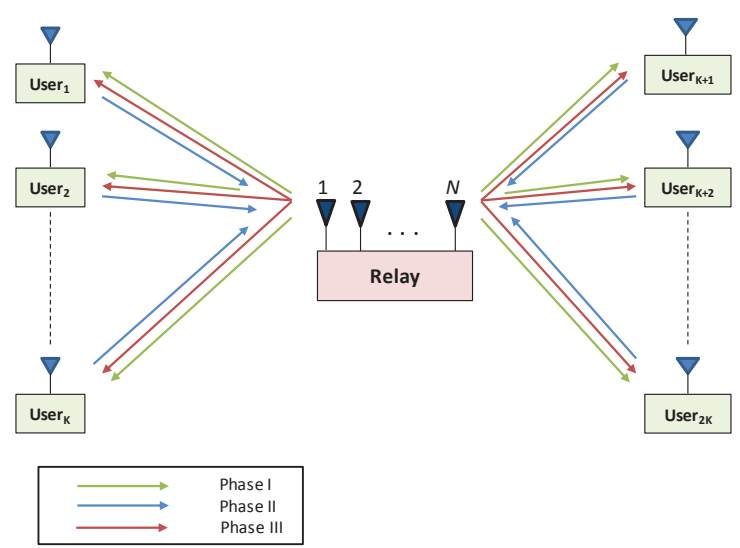

Figure 1: An energy-constrained multi-pair WPCN with multiple antenna AF relay.

are derived in two cases based on the knowledge of the channel state information (CSI) of the users at the relay in the power transfer phase , namely, 1) unknown CSI 2) partially known CSI. The contribution of this paper is twofold. We first derive analytical expressions for the ergodic spectral and energy efficiencies of the proposed system in the two cases, and confirm the analysis with computer simulations. We then examine the impact of various system parameters, such the EH time of the harvestthen-transmit protocol, number of users and relay antennas, on the system performance ${ }^{1}$.

The rest of this paper is organized as follows. Section II describes the system model under consideration. Section III derives analytical expressions for the ergodic spectral and energy efficiencies when the CSI of the users is unknown in the first phase. Section IV derives analytical expressions for the ergodic spectral and energy efficiencies when the CSI of the users is partially known in the first phase. Numerical examples and simulation results are presented and discussed in section $\mathrm{V}$. Finally, section VI draws the main conclusions.

The notations used in this paper are: Bold uppercase and bold lowercase letters denote matrices and vectors, respectively. Conjugate operation, transpose operation, conjugate transpose and inverse are denoted by $(.)^{*},(.)^{T},(.)^{H}$ and $(.)^{-1}$, respectively. The notation |.| represents the absolute value of a scalar whereas $\|$.$\| denotes Euclidean norm. Circular symmetric$ complex-Gaussian distribution is denoted by $\mathcal{C N}\left(\mu, \sigma^{2}\right)$ with mean $\mu$ and variance $\sigma^{2} . \log (),. \ln ($.$) represent logarithm of$ base-2 and base $e$, respectively. $\mathrm{I}_{N}, \mathbf{O}_{n \times m}$ and $\in \mathbb{C}^{M \times N}$ represent an $N \times N$ identity matrix, $n \times m$ all zero matrix and $M \times N$ matrix, respectively; $\operatorname{Tr}($.$) is the trace of a matrix, [A]_{k, k}$ is the element $(k, k)$ and $[A]_{k}$ is the column $k$ in matrix $A$. $\mathbb{E}$ is used for the expectation and $\operatorname{det}(\mathbf{A})$ denotes the determinant of matrix $\mathbf{A}$.

\section{SYSTEM MOdEL}

As mentioned in the introduction, we consider a WPCN consisting of $K$ pairs of single-antenna users communicating with each other via a two-way amplify and forward (AF) relay equipped with $N$ antennas as illustrated in Fig. 1, where

\footnotetext{
${ }^{1}$ This paper is a part of our work in [14] .
}

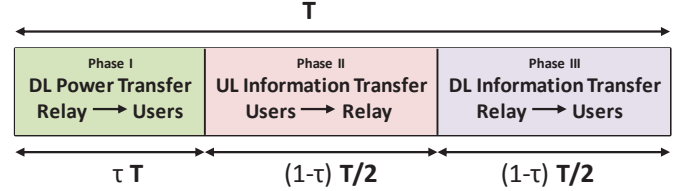

Figure 2: The harvest-then-transmit protocol.

$N>2 K$. The relay is connected to a constant power supply whereas the users are EH nodes. All the channels in this paper are modeled as independent identically distributed (i.i.d) Rayleigh fading channels. In the power transfer phase, phase I, the channel matrix between the relay and the $2 K$ users is denoted by $\mathbf{G} \in \mathbb{C}^{2 K \times N}$, which can be represented as $\mathbf{G}=\mathbf{D}^{1 / 2} \mathbf{G}_{1}$ where $\mathbf{G}_{1} \in \mathbb{C}^{2 K \times N}$ contains i.i.d $\mathcal{C N}(0,1)$ entries which represent small scale fading coefficients and $\mathbf{D} \in \mathbb{C}^{2 K \times 2 K}$ is a diagonal matrix with $[\mathbf{D}]_{k k}=\varpi_{k}$ which represent the path-loss attenuation $\varpi_{k}=d_{k}^{-m}, d_{k}$ is the distance between the relay and the $k^{t h}$ user and $m$ is the path loss exponent. The channel matrix in phase II is $\mathbf{H}_{s r} \in \mathbb{C}^{N \times 2 K}$, which can be represented as $\mathbf{H}_{s r}=\mathbf{H}_{1} \mathbf{D}^{1 / 2}$ where $\mathbf{H}_{1} \in \mathbb{C}^{N \times 2 K}$ contains i.i.d $\mathcal{C N}(0,1)$ entries and in phase III it is denoted as $\mathbf{H}_{r s} \in \mathbb{C}^{2 K \times N}$ which can also be represented as $\mathbf{H}_{r s}=\mathbf{D}^{1 / 2} \mathbf{H}_{2}$ where $\mathbf{H}_{2} \in \mathbb{C}^{2 K \times N}$ contains i.i.d $\mathcal{C N}(0,1)$ entries. Due to the poor quality of the user-to-user channel, there is no direct link between the users and all communications are managed by the relay. In addition, each user is equipped with a battery to store the harvested energy. In this respect, we adopt the harvest-then-transmit protocol introduced in [9] [11] and is shown in Fig. 2 for convenience, where $T$ is the transmission time.

To elaborate, during the first time segment $\tau T$, where $0<\tau<1$, the relay broadcasts energy signals to all users simultaneously. In the second time segment $(1-\tau) T / 2$, the users, using the harvested energy, transmit their independent signals to the relay. During the last time segment, $(1-\tau) T / 2$, the relay amplifies and forwards the information signals to their intended destinations.

In the following, the ergodic spectral and energy efficiencies are derived for two cases based on the availability of the CSI of the users at the relay in the first phase.

\section{UNKNOWN CSI}

In this case the relay is unable to know the CSI of the users in the first phase. Therefore, the received signal during the power transfer phase at the $k^{t h}$ user can be expressed as

$$
y_{s k}=\sqrt{\frac{P_{r p}}{N}} \mathbf{g}_{k} \mathbf{s}_{r}+n_{s k}
$$

where $P_{r p}$ is the relay power in phase $\mathrm{I}, \mathbf{g}_{k} \in \mathbb{C}^{1 \times N}$ is the channel vector between the relay and the $k^{t h}$ user, i.e, $k^{t h}$ vector in the matrix $\mathbf{G}^{H}, \mathbf{s}_{r} \in \mathbb{C}^{N \times 1}$ is the transmitted signal vector (energy carrying signals), energy signals are assumed to be independent and identically distributed (i.i.d.) random variables (RVs) and $n_{s k}$ is additive wight Gaussian noise (AWGN) at the $k^{t h}$ user, $n_{s k} \sim \mathcal{C N}\left(0, \sigma_{s k}^{2}\right)$. Therefore, the harvested energy by the $k^{t h}$ user, after ignoring the negligible energy harvested from the receiver noise, can be expressed as [12]

$$
E_{s k}=\eta T \tau \mathbb{E}\left[\left|y_{s k}\right|^{2}\right]=\eta T \tau\left(\frac{P_{r p}}{N}\left\|\mathbf{g}_{k}\right\|^{2}\right)
$$


where $0<\eta \leq 1$ is the EH efficiency at the receiver. The transmitted power of the $k^{t h}$ user in the second phase can be written as

$$
P_{k}=\frac{E_{s k}}{(1-\tau) T / 2}=\frac{\eta \tau\left(P_{r n}\left\|\mathbf{g}_{k}\right\|^{2}\right)}{(1-\tau) / 2}
$$

where $P_{r n}=\frac{P_{r p}}{N}$. Since $\left\|\mathbf{g}_{k}\right\|^{2}$ follows Gamma distribution, the average transmitted power can be given by

$$
\bar{P}_{k}=\frac{2 \eta \tau \varpi_{k} P_{r n} N}{(1-\tau)}
$$

The transmitted signal of user $k$ can then be expressed as

$$
x_{k}=\sqrt{P_{k}} s_{k}
$$

where $s_{k}$ is the information signal of user $k$, information signals of the users are assumed to be i.i.d. with zero mean and unit variance. The received signals at the relay in phase II can be given as

$$
\mathbf{y}_{r}=\sum_{i=1}^{2 K} \mathbf{h}_{s r_{i}} x_{i}+\mathbf{n}_{r}=\mathbf{H}_{s r} \mathbf{x}+\mathbf{n}_{r}
$$

where $\mathbf{h}_{s r_{i}}$ denotes the channel vector between the user $i$ and the relay, i.e, the $i^{t h}$ vector of the matrix $\mathbf{H}_{s r}, \mathbf{x} \in \mathbb{C}^{2 K \times 1}$ is the transmitted signal vector of all users and $\mathbf{n}_{r} \in \mathbb{C}^{N \times 1}$ is AWGN vector at the relay, $\mathbf{n}_{r} \sim \mathcal{C N}\left(0, \sigma_{r}^{2} \mathbf{I}_{N}\right)$. As mentioned above, in the third phase, the relay amplifies and forwards the received signals. Therefore, the transmitted signal vector form the relay can be expressed as

$$
\mathbf{x}_{r}=\mathbf{W} \mathbf{y}_{r}
$$

where $\mathbf{W}$ is the $N \times N$ relay weight matrix, the amplified signal $\mathbf{x}_{r}$ should satisfy the power constraint at the relay. Then the received signal at the $k^{t h}$ user can be written as

$$
\begin{aligned}
y_{k}= & \mathbf{h}_{r s_{k}} \mathbf{x}_{r}+n_{k} \\
= & \underbrace{\sqrt{P_{k^{\prime}}} \mathbf{h}_{r s_{k}} \mathbf{W} \mathbf{h}_{s r_{k^{\prime}}} s_{k^{\prime}}}_{\text {Information signal term }} \\
& +\underbrace{\sum_{i=1, i \neq k^{\prime}}^{2 K} \sqrt{P_{i}} \mathbf{h}_{r s_{k}} \mathbf{W} \mathbf{h}_{s r_{i}} s_{i}+\mathbf{h}_{r s_{k}} \mathbf{W} \mathbf{n}_{r}+n_{k}}_{\text {Interference and noise term }}
\end{aligned}
$$

where $\mathbf{h}_{r s_{k}}$ is the channel vector between the relay and the user $k$ (the $k^{t h}$ vector of the matrix $\mathbf{H}_{r s}^{H}$ ), the $k^{t h}$ and $k^{\prime}$ th users are a communication pair, i. e. $\left(k, k^{\prime}\right)=(i, K+i)$ or $(K+$ $i, i)$, where $i=1, \ldots, K$ and $n_{k}$ is the AWGN at the $k^{t h}$ user, $n_{k} \sim \mathcal{C N}\left(0, \sigma_{k}^{2}\right)$. After some basic algebraic manipulations, the signal-to-interference noise ratio (SINR) at the user $k$ can be expressed as

$$
\gamma_{k}=\frac{P_{k^{\prime}}\left|\mathbf{h}_{r s_{k}} \mathbf{W} \mathbf{h}_{s r_{k^{\prime}}}\right|^{2}}{\sum_{i=1, i \neq k^{\prime}}^{2 K} P_{i}\left|\mathbf{h}_{r s_{k}} \mathbf{W h}_{s r_{i}}\right|^{2}+\sigma_{r}^{2}\left\|\mathbf{h}_{r s_{k}} \mathbf{W}\right\|^{2}+\sigma_{k}^{2}}
$$

According to the SINR expression, the ergodic spectral efficiency of the multi-pair two-way AF relay system can be given by

$$
R=\mathbb{E}\left[\frac{(1-\tau)}{2} \sum_{i=1}^{2 K} \log \left(1+\gamma_{i}\right)\right]
$$

In addition, the energy efficiency of a multi-pair two-way relaying system, is defined as the ergodic spectral efficiency, $R$, divided by the total transmit power, i. e. [15]

$$
\rho=\frac{R}{P_{t}}
$$

where $P_{t}$ is the total power. In order to improve the system performance ZFR/ZFT is implemented at the relay as described in the next section.

\section{A. Analysis of Ergodic Spectral and Energy Efficiencies}

According to [13], [15], the ZFR/ZFT matrix at the relay can be given as

$$
\begin{aligned}
& \mathbf{W}=\beta \mathbf{W}_{z f} \\
& =\beta\left(\mathbf{H}_{r s}^{H}\right)\left(\mathbf{H}_{r s} \mathbf{H}_{r s}^{H}\right)^{-1} \Pi\left(\mathbf{H}_{s r}^{H} \mathbf{H}_{s r}\right)^{-1}\left(\mathbf{H}_{s r}^{H}\right)
\end{aligned}
$$

where $\Pi$ is the permutation matrix to represent the user-pairing format and takes the form of $\left[\mathbf{O}_{K \times K}, \mathbf{I}_{K} ; \mathbf{I}_{K}, \mathbf{O}_{K \times K}\right]$ [13], $\beta$ is the amplification gain to satisfy the relay power constraint. According to (6) and (7) we can write $\beta$ as in

$$
\beta=\sqrt{\frac{P_{r i}}{\operatorname{Tr}\left(\mathbb{E}\left[\mathbf{W}\left(\mathbf{H}_{s r} \Theta \mathbf{H}_{s r}^{H}+\mathbf{n}_{r} \mathbf{n}_{r}^{H}\right) \mathbf{W}^{H}\right]\right)}}
$$

where $P_{r i}$ is the relay power in phase III and $\Theta=\mathrm{E}\left(\mathbf{x x}^{\mathbf{H}}\right)$. Substituting (12) into (13) yields

$$
\beta=\sqrt{\frac{P_{r i}}{Q+\sigma_{r}^{2} Q^{\prime}}}
$$

where $Q=\operatorname{Tr}\left(\mathbb{E}\left[\left(\mathbf{H}_{r s} \mathbf{H}_{r s}^{H}\right)^{-1} \Theta\right]\right)$ and $Q^{\prime}=$ $\operatorname{Tr}\left(\mathbb{E}\left[\left(\mathbf{H}_{r s}^{H} \mathbf{H}_{s r}^{H} \mathbf{H}_{s r} \mathbf{H}_{r s}\right)^{-1}\right]\right)$, which are provided in [16, Lemma 1] [13]. At high signal to noise ratio (SNR) this relation can be reduced to [17]

$$
\beta=\sqrt{\frac{P_{r i}}{Q}}
$$

where $Q=\frac{\sum_{i=1}^{2 K}\left(\frac{\bar{P}_{i}}{\bar{\varpi}_{i}}\right)}{N-2 K}$. Based on the fact that $\mathbf{h}_{r s_{k}} \mathbf{W} \mathbf{h}_{s r_{i}}=\beta \delta$, where $\delta=1$ when $i=k^{\prime}$ and $\delta=0$ otherwise, we can now rewrite the received signal at the $k^{\text {th }}$ user as follows

$$
y_{k}=\beta \sqrt{P_{k^{\prime}}} s_{k^{\prime}}+\mathbf{h}_{r s_{k}} \mathbf{W} \mathbf{n}_{r}+n_{k}
$$

Comparing (8) and (16), it is is clear that the ZFR/ZFT scheme eliminated the interference. At this stage, the SINR in (9) can be rewritten as

$$
\gamma_{k}=\frac{P_{k^{\prime}} \beta^{2}}{\sigma_{r}^{2} \beta^{2}\left[\left(\mathbf{H}_{s r}^{H} \mathbf{H}_{s r}\right)^{-1}\right]_{k^{\prime}, k^{\prime}}+\sigma_{k}^{2}}
$$


Substituting (3) into (17) yields

$$
\gamma_{k}=\frac{\frac{\eta \tau\left[P_{r n}\left\|\mathbf{g}_{k^{\prime}}^{H}\right\|^{2}\right]}{(1-\tau) / 2} \beta^{2}}{\sigma_{r}^{2} \beta^{2}\left[\left(\mathbf{H}_{s r}^{H} \mathbf{H}_{s r}\right)^{-1}\right]_{k^{\prime}, k^{\prime}}+\sigma_{k}^{2}}
$$

which can also be written as

$$
\gamma_{k}=\frac{a_{1}\left\|\mathbf{g}_{k^{\prime}}^{H}\right\|^{2}}{a_{2}\left[\left(\mathbf{H}_{s r}^{H} \mathbf{H}_{s r}\right)^{-1}\right]_{k^{\prime}, k^{\prime}}+a_{3}}
$$

where

$$
\begin{aligned}
& a_{1}=2 \beta^{2} \eta \tau P_{r n} \\
& a_{2}=\beta^{2}(1-\tau) \sigma_{r}^{2}, \text { and } \\
& a_{3}=\sigma_{k}^{2}(1-\tau) .
\end{aligned}
$$

Now substituting $X=a_{1}\left\|\mathbf{g}_{k^{\prime}}^{H}\right\|^{2}$ and $Y=$ $a_{2}\left[\left(\mathbf{H}_{s r}^{H} \mathbf{H}_{s r}\right)^{-1}\right]_{k^{\prime}, k^{\prime}}+a_{3}$, the spectral efficiency in (10) can be calculated as

$$
R=(1-\tau) K \mathbb{E}\left[\log \left(1+\frac{X}{Y}\right)\right]
$$

Lemma 1. It is found in [18] that for any $u, v>0$

$$
\mathbb{E}\left[\ln \left(1+\frac{u}{v}\right)\right]=\int_{0}^{\infty} \frac{1}{z}\left(\mathcal{M}_{v}(z)-\mathcal{M}_{v, u}(z)\right) d z
$$

where $\mathcal{M}_{v}(z)$ denotes the moment generating function (MGF) of $v$ and is defined as $\mathcal{M}_{v}(z)=\mathbb{E}\left[e^{-z v}\right]$ and $\mathcal{M}_{v, u}(z)$ is defined as $\mathcal{M}_{v, u}(z)=\mathbb{E}\left[e^{-z(v+u)}\right]$.

Using this definition, and since $X$ and $Y$ are independent, (21) can be written as

$$
R=\frac{(1-\tau) K}{\ln (2)} \int_{0}^{\infty} \frac{1}{z}\left(1-\mathcal{M}_{X}(z)\right) \mathcal{M}_{Y}(z) d z
$$

where $\mathcal{M}_{X}(z)$ and $\mathcal{M}_{Y}(z)$ are the MGFs of $X$ and $Y$, receptively. Since $X$ follows Gamma distribution, its MGF can be represented as

$$
\mathcal{M}_{X}(z)=\left(1+a_{1} \varpi_{k^{\prime}} z\right)^{-N}
$$

where $\varpi_{k^{\prime}}$ is the the $k^{\prime}$ th diagonal element of $\mathbf{D}$. Probability distribution function (PDF) of $\zeta=\left[\left(\mathbf{H}_{s r}^{H} \mathbf{H}_{s r}\right)^{-1}\right]_{k^{\prime}, k^{\prime}}$ is [13]

$$
f_{\zeta}(y)=\frac{\left(\Psi_{k^{\prime}}\right)^{N-2 K+1} e^{\frac{-\Psi_{k^{\prime}}}{y}}}{\Gamma(N-2 K+1) y^{(N-2 K+2)}}
$$

where $\Psi_{k^{\prime}}$ is the $k^{\prime}$ th diagonal element of $\mathbf{D}^{-1}$. Then, the MGF of $\zeta$ can be calculated as

$$
\mathcal{M}_{\zeta}(z)=\int_{0}^{\infty} e^{-z y} f_{\zeta}(y) d y
$$

Now, using the identities in [19], [20], we can find the MGF of $Y$ as

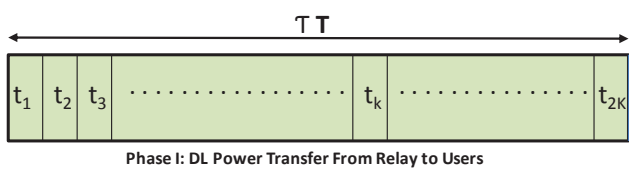

Figure 3: The antenna selection protocol.

$\mathcal{M}_{Y}(z)=\frac{2 e^{-a_{3} z}\left(a_{2} \Psi_{k^{\prime}} z\right)^{\frac{N-2 K+1}{2}} \mathrm{~J}\left(N-2 K+1,2 \sqrt{a_{2} \Psi_{k^{\prime}} z}\right)}{\Gamma(N-2 K+1)}$

where $\mathbf{J}($.$) is the modified Bessel function of the second kind$ [20]. Finally, substituting (24) and (27) into (23) yields the system spectral efficiency as in (28), shown at the top of the next page. The corresponding energy efficiency is simply calculated by $(11)$.

\section{PARTIAL CSI}

In this case the relay has the ability to know the amplitude of the users' channels in the first phase. Therefore, in order to maximize the received energy signals at the users in the first phase, antenna selection (AS) scheme is proposed in this section. In this scheme, the time segment allocated to charge the users $\tau T$ is divided into equal $2 K$ time slots, in each slot the best antenna which has best channel to a user is selected to transmit the energy signal with full relay power $P_{r p}$, i.e, in the first time slot $t_{1}$ the best antenna for user 1 is selected, in the second time slot $t_{2}$ the best antenna for user 2 is selected and so on, as shown in Fig 3. Consequently, user $k$ will receive energy signals in all the period $\tau T$, but in the time slot $t_{k}$ receives the energy signal from its best antenna and during the remaining time $\tau T-t_{k}$ receives the energy signal from one of the $N$ antennas based on the selection of the other users.

Therefore, the received energy signal during the power transfer phase at the $k^{t h}$ user can be expressed as

$y_{s k}=\underbrace{\sqrt{P_{r p}} g_{i} s_{r}}_{t_{1}}+\ldots \ldots+\underbrace{\sqrt{P_{r p}} g_{k}^{*} s_{r}}_{t_{k}}+\ldots \ldots+\underbrace{\sqrt{P_{r p}} g_{r} s_{r}}_{t_{2 K}}+n_{s k}$

where $g_{i}$ is the channel between one of the relay antennas (based on the selection of the first user) and the $k^{\text {th }}$ user, $g_{k}^{*}$ is the best channel between the relay and the $k^{t h}$ user, $g_{r}$ is the channel between one of the relay antennas (based on the selection of the $2 K^{t h}$ user) and the $k^{t h}$ user and $s_{r}$ is the energy signal. The harvested energy by the $k^{t h}$ user can be expressed as

$E_{s k}=\eta T\left(t_{1} P_{r p}\left|g_{i}\right|^{2}+\ldots .+t_{k} P_{r p}\left|g_{k}^{*}\right|^{2}+\ldots+t_{2 K} P_{r p}\left|g_{k}\right|^{2}\right)$

where $t_{i}=\frac{\tau}{2 K}$. we can write the harvested energy as

$E_{s k}=\eta T\left(t_{k} P_{r p}\left|g_{k}^{*}\right|^{2}+t_{1} P_{r p} \sum_{i=1}^{N}\left|g_{i}\right|^{2} \operatorname{Pr}(\right.$ antenna selected $=i)+\ldots$

$\ldots .+t_{2 K} P_{r p} \sum_{i=1}^{N}\left|g_{i}\right|^{2} \operatorname{Pr}($ antenna selected $\left.=i)\right)$ 


$$
R=\frac{(1-\tau) K}{\ln (2)} \int_{0}^{\infty}\left(\frac{1}{z}\left(1-\left(1+a_{1} \varpi_{k^{\prime}} z\right)^{-N}\right) \frac{2 e^{-a_{3} z}\left(a_{2} \Psi z\right)^{\frac{N-2 K+1}{2}} \mathrm{~J}\left(N-2 K+1,2 \sqrt{a_{2} \Psi z}\right)}{\Gamma(N-2 K+1)}\right) d z
$$

which can be simplified as

$$
E_{s k}=\eta T\left(\frac{\tau}{2 K} P_{r p}\left|g_{k}^{*}\right|^{2}+\frac{\tau(2 K-1)}{2 K} P_{r p} \sum_{i=1}^{N}\left|g_{i}\right|^{2} \frac{1}{N}\right)
$$

The harvested power for the $k^{t h}$ user can be given as

$$
P_{k}=\frac{E_{s k}}{(1-\tau) T / 2}=\frac{\eta P_{r p} \frac{\tau}{2 K}\left(\left|g_{k}^{*}\right|^{2}+\frac{(2 K-1)}{N} \sum_{i=1}^{N}\left|g_{i}\right|^{2}\right)}{(1-\tau) / 2}
$$

Since $\left|g_{i}\right|^{2}$ has exponential distribution and $\left|g_{k}^{*}\right|^{2}$ is the maximum of exponential random variables (RVs), the average harvested power can be given by [21]

$$
\bar{P}_{k}=\frac{\eta \varpi_{k} \tau P_{r p}\left(\sum_{i=1}^{N}\left(\frac{1}{i}\right)+(2 K-1)\right)}{(1-\tau) K}
$$

The transmitted signal of the user $k$ can then be expressed as

$$
x_{k}=\sqrt{P_{k}} s_{k}
$$

where $s_{k}$ is the information signal of the user $k$, information signals of the users are assumed to be i.i.d. with zero mean and unit variance.

\section{A. Analysis of Ergodic Spectral and Energy Efficiencies}

Substituting (33) into (17), the SINR becomes

$$
\gamma_{k}=\frac{\left(\frac{\eta \beta^{2} \tau P_{r p} \varpi_{k}}{(1-\tau) K}\right)\left(\left|g_{1 k^{\prime}}^{*}\right|^{2}+\frac{(2 K-1)}{N} \sum_{i=1}^{N}\left|g_{1 i^{\prime}}\right|^{2}\right)}{\sigma_{r}^{2} \beta^{2}\left[\left(\mathbf{H}_{s r}^{H} \mathbf{H}_{s r}\right)^{-1}\right]_{k^{\prime}, k^{\prime}}+\sigma_{k}^{2}}
$$

where $\left|g_{1 k^{\prime}}^{*}\right|^{2}=\varpi_{k}\left|g_{k^{\prime}}^{*}\right|^{2}$ and $\left|g_{1 i^{\prime}}\right|^{2}=\varpi_{k}\left|g_{i^{\prime}}\right|^{2}$. The SINR can also be written as

$$
\gamma_{k}=\frac{\left|g_{1 k^{\prime}}^{*}\right|^{2}+\frac{(2 K-1)}{N} \sum_{i=1}^{N}\left|g_{1 i^{\prime}}\right|^{2}}{\left(\frac{a_{2}}{a_{1}}\right)\left[\left(\mathbf{H}_{s r}^{H} \mathbf{H}_{s r}\right)^{-1}\right]_{k^{\prime}, k^{\prime}}+\left(\frac{a_{3}}{a_{1}}\right)}
$$

where

$$
\begin{aligned}
& a_{1}=\varpi_{k} \beta^{2} \eta \tau P_{r p} /(1-\tau) K \\
& a_{2}=\beta^{2} \sigma_{r}^{2}, \text { and } \\
& a_{3}=\sigma_{k}^{2} .
\end{aligned}
$$

Now substituting $X=\left|g_{1 k^{\prime}}^{*}\right|^{2}, \chi=\frac{(2 K-1)}{N} \sum_{i=1}^{N}\left|g_{1 i^{\prime}}\right|^{2}$ and $Y=\left(\frac{a_{2}}{a_{1}}\right)\left[\left(\mathbf{H}_{s r}^{H} \mathbf{H}_{s r}\right)^{-1}\right]_{k^{\prime}, k^{\prime}}+\left(\frac{a_{3}}{a_{1}}\right)$ and using the definition in lemma 1 , the ergodic spectral efficiency can be written as

$$
R=\frac{(1-\tau) K}{\ln (2)} \int_{0}^{\infty} \frac{1}{z}\left(1-\mathcal{M}_{X, \chi}(z)\right) \mathcal{M}_{Y}(z) d z
$$

According to the order statistics of exponential RVs, the PDF of the maximum of exponential RVs is given by [21]

$$
f_{X}(x)=N e^{-x}\left(1-e^{-x}\right)^{N-1}
$$

Therefore, $\mathcal{M}_{X}(z)$ can be derived as

$$
\mathcal{M}_{X}(z)=\frac{N \Gamma(N) \Gamma(1+z)}{\Gamma(1+N+z)}
$$

and $\mathcal{M}_{\chi}(z)$ is the MGF of Chi-square RV given by

$$
\mathcal{M}_{\chi}(z)=\left(1+\frac{2(2 K-1)}{N} z\right)^{\frac{-N}{2}}
$$

Similarly as in the previous section, we can find the MGF of $Y$ as in (43)- at the top of the next page.

Substituting (41), (42) and (43) into (39) we can get the ergodic spectral efficiency as in (44)- at the top of the next page. The corresponding energy efficiency is simply calculated by (11).

\section{Numerical Results AND Discussions}

In this section we present some numerical results of the analytical expressions derived above. Monte Carlo simulations with $10^{6}$ independent trials are conducted in which channel coefficients are randomly generated in each simulation run and the noise power at all nodes is set as $\sigma_{r}^{2}=\sigma_{k}^{2}=0 \mathrm{dBw}$. Unless it is mentioned otherwise, $\varpi$ and $\eta^{2}$ are chosen to be 1 .

\section{A. Effect of EH Time $\tau$}

To demonstrate the impact of EH time on the system performance, we plot in Fig. 4 the spectral efficiency versus $\tau$ for various values of $N$ when $K=3, P_{r n}=0.4 \mathrm{~W}$. The good agreement between the analytical and simulated results confirms the validity of our analysis in the previous sections. It should be pointed out that the analytical results are obtained from (28) and (44). It is evident from these results that the ergodic spectral efficiency enhances with increasing the number of relay antennas. This is because increasing $N$ implies increasing the amount of energy arrived at each user which eventually leads to increasing their transmitted power. The other observation is that, for each value of $N$ there exists an optimal $\mathrm{EH}$ time $\left(\tau^{*}\right)$ that maximizes the spectral efficiency. It is noted that $\tau^{*}$ becomes smaller when $N$ is increased. This is justified by the fact that, increasing $N$ means increasing the relay transmitted power during phase $\mathrm{I}$ and therefore the users require smaller amount of EH time to attain optimal performance. Finally, and

${ }^{2}$ Practically speaking, although it is true that this value is not realistic and is always less than one, same trends will be observed for smaller $\eta$ values as will be shown later. 


$$
\mathcal{M}_{Y}(z)=\frac{2 e^{-\left(\frac{a_{3}}{a_{1}}\right) z}\left(\left(\frac{a_{2}}{a_{1}}\right) \Psi_{k^{\prime}} z\right)^{\frac{N-2 K+1}{2}} \mathrm{~J}\left(N-2 K+1,2 \sqrt{\left.\left(\frac{a_{2}}{a_{1}}\right) \Psi_{k^{\prime}} z\right)}\right.}{\Gamma(N-2 K+1)}
$$

$$
\begin{gathered}
R=\frac{(1-\tau) K}{\ln (2)} \int_{0}^{\infty} \frac{1}{z}(1-\underbrace{\frac{N \Gamma(N) \Gamma(1+z)}{\Gamma(1+N+z)}\left(1+\frac{2(2 K-1)}{N} z\right)^{\frac{-N}{2}}}_{\mathcal{M}_{X}(z)}) \\
(\underbrace{\left.\frac{2 e^{-\left(\frac{a_{3}}{a_{1}}\right) z}\left(\left(\frac{a_{2}}{a_{1}}\right) \Psi z\right)^{\frac{N-2 K+1}{2}} \mathrm{~J}\left(N-2 K+1,2 \sqrt{\left(\frac{a_{2}}{a_{1}}\right) \Psi z}\right)}{\Gamma(N-2 K+1)}\right)}_{\mathcal{M}_{\chi}(z)}) d z
\end{gathered}
$$

as expected, the ergodic spectral efficiency in partial CSI case is higher than that in unknown CSI.

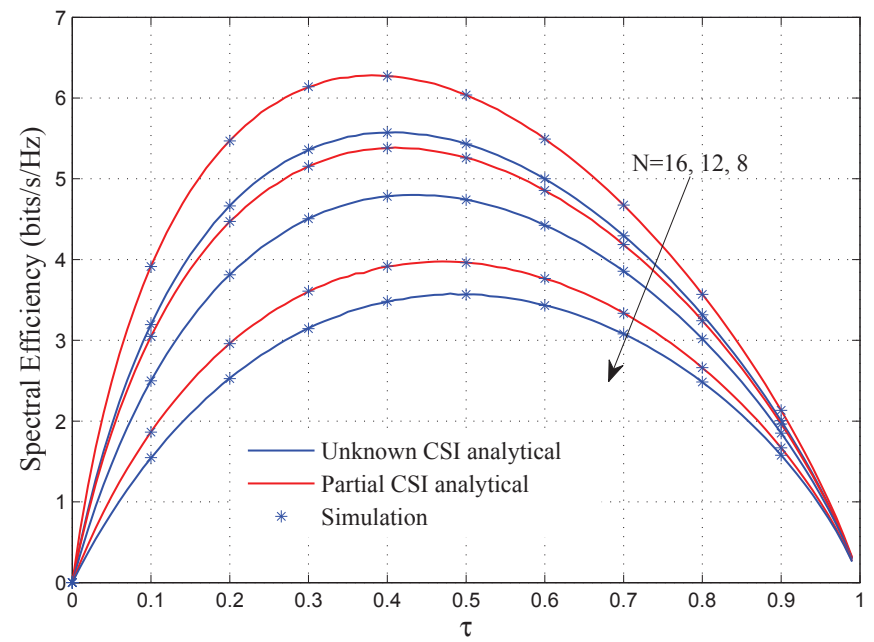

Figure 4: Ergodic spectral efficiency versus $\tau$ for various values of $N$.

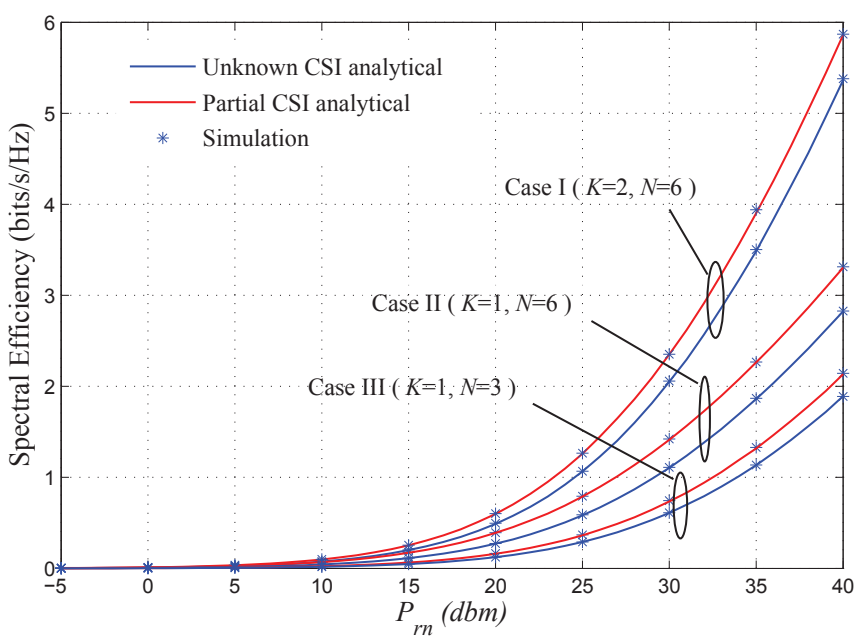

Figure 5: Ergodic spectral efficiency against per antenna relay power for different values of $K$ and $N$.

\section{B. Effect of The Number of Pairs and Relay Power on Spectral} Efficiency

In this subsection, we capture the influence of the number of user-pairs and the relay power in phase I on the system performance. Therefore, we present in Fig. 5 the spectral efficiency versus per antenna relay power $P_{r n}$ for three different combinations of $K$ and $N$, case I $(K=2, N=6)$, case II $(K=1, N=6)$ and case III $(K=1, N=3)$, when $\eta=0.4$. In general, we can notice that increasing the relay power in phase I improves the spectral efficiency for all the studied combinations. In addition, comparing the cases I and II for each scheme, it can be seen that, for a given value of $N$, the system performance enhances as $K$ is increased and this enhancement becomes larger with increasing the relay power. On the other hand, comparing the cases II and III for each scheme, it is interesting to notice that the gain attained with doubling $N$ is independent of the relay power. It is also apparent that, partial CSI scheme outperforms the unknown CSI scheme in the three combinations.

\section{Effect of Number of Relay Antennas, EH efficiency and Number of Pairs on Energy Efficiency}

The impact of the number of relay antennas, the EH efficiency and the number of pairs on the maximum achievable energy efficiency of the proposed system are investigated in this subsection $^{3}$. Fig. 6a shows some numerical and simulated results of the energy efficiency as a function of the number of relay antennas for several values of $\eta$ when $P_{r i}=1 \mathrm{~W}, P_{r p}=3 \mathrm{~W}$ and $K=4$. It can be seen that increasing the number of relay antennas always improves the energy efficiency irrespective of the values of $\eta$ and this enhancement is more significant in partial CSI scheme. Notably, and as anticipated, reducing the EH efficiency degrades the system performance.

Fig. $6 \mathrm{~b}$ shows the energy efficiency versus the number of relay antennas $N$ when $K=1$ and $K=2$. From the figure we can observe that, when the number of relay antennas is small $N<4$ the case of $K=1$ has larger energy efficiency than that of

\footnotetext{
${ }^{3}$ Maximum achievable energy efficiency implies the energy efficiency of the
} optimized system, i.e. when the EH time is optimized. 


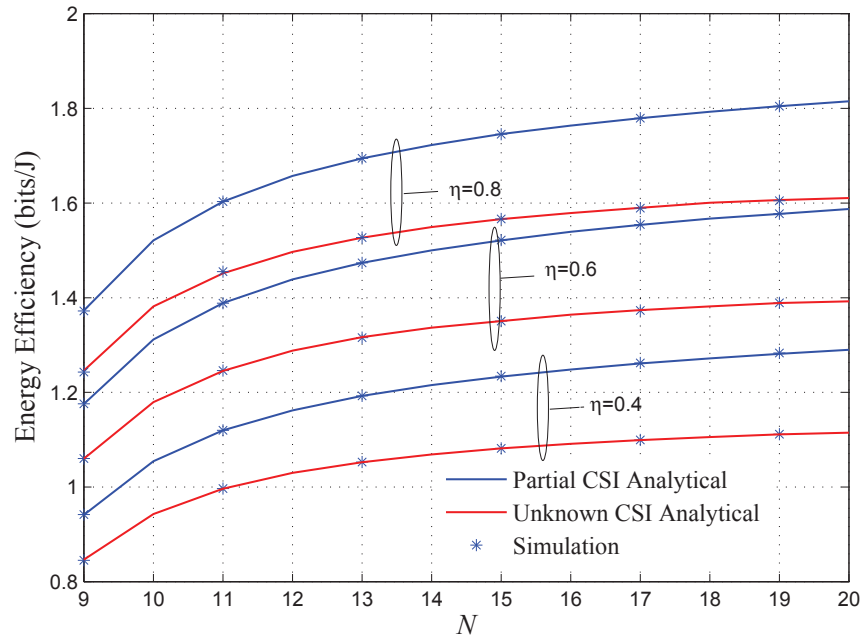

(a) Energy efficiency versus $N$ for various values of $\eta$.

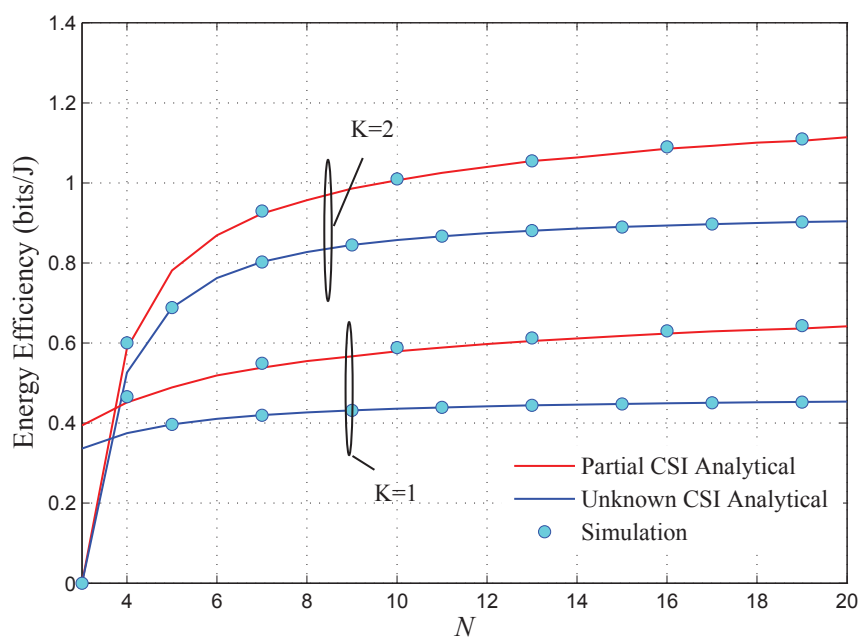

(b) Energy efficiency versus $N$ for various values of $K$.

Figure 6: Effect of Number of Relay Antennas and EH efficiency/Number of Pairs on Energy Efficiency.

$K=2$, and this is because the interference at the relay can not be reduced when the number of the relay antennas is smaller than $2 K$. On the other hand, when the number of the relay antennas increases $N>4$ the energy efficiency of $K=2$ becomes greater than that of $K=1$.

\section{CONCLUSION}

In this paper, we have analyzed a wireless powered communication network with $N$-antennas two-way AF relay and singleantenna $K$-pair users. The spectral and energy efficiencies are investigated when the ZFR/ZFT is applied at the relay node in two cases, when the CSI is un-known/partially-known in phase I. We have derived analytical expressions for the spectral and energy efficiencies, which are then validated with Monte Carlo simulations. The impact of some system parameters, such as $\mathrm{EH}$ time parameter, EH efficiency, number of users and relay antennas, on the spectral and energy efficiencies of the proposed system were investigated. It was shown that a good selection of the EH time is the key for achieving optimal performance.

\section{REFERENCES}

[1] L. Varshney, "Transporting information and energy simultaneously," in Proc. Int. Sympos. Inf. Theory (ISIT), pp. 1612-1616, Jul. 2008.

[2] P. Grover and A. Sahai, "Shannon meets tesla: Wireless information and power transfer," in Proc. Int. Sympos. Inf. Theory (ISIT), pp. 2363-2367, Jun. 2010.

[3] X. Zhou, R. Zhang, and C. K. Ho, "Wireless information and power transfer: Architecture design and rate-energy tradeoff," IEEE Trans. Commun., vol. 61, pp. 4754-4767, Nov. 2013.

[4] L. Liu, R. Zhang, and K.-C. Chua, "Wireless information transfer with opportunistic energy harvesting," IEEE Trans. Wireless Commun., vol. 12, pp. 288-300, Jan. 2013.

[5] Z. Xiang and M. Tao, "Robust beamforming for wireless information and power transmission," IEEE Wireless Commun. Lett., vol. 1, pp. 372-375, Aug. 2012.

[6] R. Zhang and C. K. Ho, "MIMO broadcasting for simultaneous wireless information and power transfer," IEEE Trans. Wireless Commun., vol. 12 pp. 1989-2001, May 2013.
[7] B. Medepally and N. Mehta, "Voluntary energy harvesting relays and selection in cooperative wireless networks," IEEE Trans. Wireless Commun., vol. 9, pp. 3543-3553, Nov. 2010 .

[8] W. Huang, H. Chen, Y. Li, and B. Vucetic, "On the performance of multiantenna wireless-powered communications with energy beamforming," IEEE Trans. Veh. Technol., vol. PP, no. 99, pp. 1-1, 2015.

[9] H. Chen, Y. Li, J. Luiz Rebelatto, B. Uchoa-Filho, and B. Vucetic, "Harvest-then-cooperate: Wireless-powered cooperative communications," Signal Processing, IEEE Transactions on, vol. 63, pp. 1700-1711, April 2015.

[10] G. Moritz, J. Rebelatto, R. Demo Souza, B. Uchoa-Filho, and Y. Li, "Timeswitching uplink network-coded cooperative communication with downlink energy transfer," IEEE Trans. Signal Process., vol. 62, pp. 5009-5019, Oct. 2014.

[11] H. Ju and R. Zhang, "Throughput maximization in wireless powered communication networks," IEEE Trans. Wireless Commun., vol. 13, pp. 418428, Jan. 2014

[12] L. Liu, R. Zhang, and K.-C. Chua, "Multi-antenna wireless powered communication with energy beamforming," IEEE Trans. Commun., vol. 62, pp. 4349-4361, Dec. 2014

[13] G. Amarasuriya, C. Tellambura, and M. Ardakani, "Sum rate analysis of two-way MIMO AF relay networks with zero-forcing," IEEE Trans. Wireless Commun., vol. 12, pp. 4456-4469, Sept. 2013.

[14] A. Salem and K. A. Hamdi, "Wireless power transfer in multi-pair two-way af relaying networks," in submitted to TCOM IEEE, IEEE Trans. Commun.

[15] H. Cui, L. Song, and B. Jiao, "Multi-pair two-way amplify-and-forward relaying with very large number of relay antennas," IEEE Trans. Wireless Commun., vol. 13, pp. 2636-2645, May 2014.

[16] R. Wang and M. Tao, "Outage performance analysis of two-way relay system with multi-antenna relay node," in Communications (ICC), 2012 IEEE International Conference on, pp. 3538-3542, June 2012.

[17] R. H. Y. Louie, Y. Li, and B. Vucetic, "Zero forcing in general two-hop relay networks," IEEE Trans. Veh. Technol., vol. 59, pp. 191-202, Jan. 2010

[18] K. Hamdi, "A useful lemma for capacity analysis of fading interference channels," IEEE Trans. Commun., vol. 58, pp. 411-416, Feb. 2010.

[19] S. M. ROSS, Introduction to probability models 10ed. 2010.

[20] I. S. Gradshteyn and I. M. Ryzhik, Table of Integrals, Series, and Products. 1980

[21] H. David., Order Statistics. 1970. 